\title{
A simple hierarchical model for heterogeneity in the evolutionary correlation on a phylogenetic tree
}

\author{
${ }_{4}$ Liam J. Revell $^{1,2}$, Ken S. Toyama ${ }^{3}$, and D. Luke Mahler ${ }^{3}$ \\ ${ }_{5}{ }^{1}$ Department of Biology, University of Massachusetts Boston, Boston, Massachusetts \\ 02125 \\ ${ }^{2}$ Facultad de Ciencias, Universidad Católica de la Santísima Concepción, Concepción, \\ Chile \\ ${ }^{3}$ Department of Ecology \& Evolutionary Biology, University of Toronto, Toronto, Ontario \\ M5S 3B2, Canada \\ Corresponding author: \\ Liam J. Revell ${ }^{1,2}$ \\ Email address: liam.revell@umb.edu
}

\begin{abstract}
Numerous questions in phylogenetic comparative biology revolve around the correlated evolution of two or more phenotypic traits on a phylogeny. In many cases, it may be sufficient to assume a constant value for the evolutionary correlation between characters across all the clades and branches of the tree. Under other circumstances, however, it's desirable or necessary to account for the possibility that the evolutionary correlation differs through time or in different sections of the phylogeny. Here, we present a method designed to fit a hierarchical series of models for heterogeneity in the evolutionary rates and correlation of two quantitative traits on a phylogenetic tree. We apply the method to two datasets: one for different attributes of the buccal morphology in sunfishes (Centrarchidae); and a second for overall body length and relative body depth in rock- and non rock-dwelling South American iguanian lizards. We also examine the performance of the method for model selection using a small set of numerical simulations.
\end{abstract}

\section{INTRODUCTION}

The evolutionary correlation is defined as the tendency of two phenotypic characteristics to co-evolve over evolutionary time or on a phylogenetic tree (Felsenstein, 1985; Revell and Collar, 2009; O'Meara, 2012; Harmon, 2019). Lots of hypotheses about evolution that are tested using phylogenetic comparative methods involve the evolutionary correlation between traits. For instance, when Garland et al. (1992) tested the hypothesis of a correlation between phylogenetically independent contrasts (Felsenstein, 1985) for home range size and body mass in mammals, they were really asking if evolutionary increases in body size tend to be associated with increases in home range size, as well as the converse. In effect, they asked if the two traits were evolutionarily correlated. Likewise, when Ruiz-Robleto and Villar (2005) used phylogenetic contrasts to explore the relationship between relative growth rate and leaf longevity in woody plants, they were in fact investigating the tendency of these two traits to co-evolve on the phylogeny. They were measuring the evolutionary correlation between different phenotypic characteristics of plant leaves (Ruiz-Robleto and Villar, 2005).

Most analyses of the evolutionary correlation assume that the tendency of traits to co-evolve is constant over all of the branches and clades of the phylogeny. Revell and Collar (2009), however, proposed a new (at the time) likelihood-based method for testing a hypothesis of a discrete shift in the evolutionary correlation or correlations between two or more traits in certain predefined parts of the phylogeny. According to this method, which was a relatively simple multivariate extension of an important related approach by O'Meara et al. (2006; also see Thomas et al., 2006; Revell and Harmon, 2008), the rate of evolution for individual traits, and the evolutionary correlation between them, were free to vary between different regimes mapped onto the phylogeny by the user. Revell and Collar (2009) applied the method to 
a phylogeny and dataset for the buccal morphology of sunfishes (Centrarchidae) to test the hypothesis that the evolutionary tendency of gape width and buccal length to co-evolve was affected by feeding mode.

Revell and Collar's (2009) approach is implemented in the phytools R package (Revell, 2012) and has been applied to various questions since its original publication. For instance, Damian-Serrano et al. (2021) used the method to test whether the the evolutionary correlation between different aspects of the prey capture apparatus in siphonophore hydrozoans changes as a function of the type of prey they consume. The method has also been updated or adapted in different ways (e.g., Clavel et al., 2015; Caetano and Harmon, 2017). For example, Clavel et al. (2015) developed software for modeling multivariate evolution, but with different types of constraints on the evolutionary rates or correlations between traits; and Caetano and Harmon (2017) implemented an extension of Revell and Collar (2009) that uses Bayesian MCMC for estimation, instead of maximum likelihood.

The underlying model of Revell and Collar (2009) is multivariate Brownian motion (Felsenstein, 1985; Revell and Harmon, 2008; Harmon, 2019). Brownian motion is a continuous time stochastic diffusion process in which the variance that accumulates between diverging lineages is proportional to the time since they shared a common ancestor (O'Meara et al., 2006; Revell and Harmon, 2008; Revell, 2021). The amount of covariance between species related by the tree is a direct function of the distance above the root of their most recent ancestor. At the tips of the tree, species values for a trait, $x$, are anticipated to have a multivariate normal distribution with a mean equal to the value at the root node of the phylogeny, and a variance-covariance matrix equal to $\sigma^{2} \mathbf{C}$ in which $\mathbf{C}$ is an $n \times n$ matrix (for $n$ total taxa) that contains the height above the root node of the common ancestor of each $i, j$ th species pair for $i \neq j$; and the total length of the tree from the root to each $i$ th tip, otherwise (O'Meara et al., 2006; Revell et al., 2008).

In the case of multivariate Brownian motion, the diffusion process can no longer be described by a single parameter, $\sigma^{2}$. Now, Brownian motion evolution is governed by an $m \times m$ matrix, for $m$ traits, sometimes referred to as the evolutionary rate matrix (Revell and Harmon, 2008; Revell and Collar, 2009; Caetano and Harmon, 2017). An example of a simple, $2 \times 2$ Browian evolutionary rate matrix is given by equation (1).

$$
\mathbf{R}=\left[\begin{array}{cc}
\sigma_{1}^{2} & \sigma_{1,2} \\
\sigma_{2,1} & \sigma_{2}^{2}
\end{array}\right]
$$

In this expression, $\sigma_{1}^{2}$ and $\sigma_{2}^{2}$ are the instantaneous variances, or Brownian motion rates (O'Meara et al., 2006), for traits 1 and 2, respectively. Meanwhile $\sigma_{1,2}$ (and $\sigma_{2,1}$ - which always has the same value; i.e., $\mathbf{R}$ is a symmetric matrix) is the instantaneous covariance of the traits 1 and 2 (Revell and Harmon, 2008). The evolutionary correlation between traits 1 and 2, in turn, is calculated as follows.

$$
r=\frac{\sigma_{1,2}}{\sqrt{\sigma_{1}^{2} \sigma_{2}^{2}}}
$$

Alternatively then, of course, equation (1) can be recomposed and expressed uniquely in terms of $r$, $\sigma_{1}$, and $\sigma_{2}$.

$$
\mathbf{R}=\left[\begin{array}{cc}
\sigma_{1}^{2} & r \sigma_{1} \sigma_{2} \\
r \sigma_{2} \sigma_{1} & \sigma_{2}^{2}
\end{array}\right]
$$

The primary innovation of Revell and Collar (2009), as well as related methods (such as Adams, 2013; Clavel et al., 2015; Caetano and Harmon, 2017), was to permit the instantaneous evolutionary variances and covariances of the Brownian motion process to differ in different parts of the tree that had been specified a priori by the investigator. Figure 1 shows just this type of analysis for a phylogeny of Centrarchidae (sunfishes), a discrete pair of evolutionary regimes (feeding mode: piscivorous or non-piscivorous), and a quantitative phenotypic trait dataset comprised of two different attributes of the feeding morphology: relative gape width and relative buccal length (Collar et al., 2005; Revell and Collar, 2009). Panel 1a gives the phylogeny with a hypothesis about how the evolutionary regime (feeding mode) may have evolved on the tree. Panel $1 \mathrm{~b}$ shows the phylogeny projected into the trait space. Finally, panel 1c shows the results of fitted one and two Brownian evolutionary rate matrix models (Figure 1). 
a)

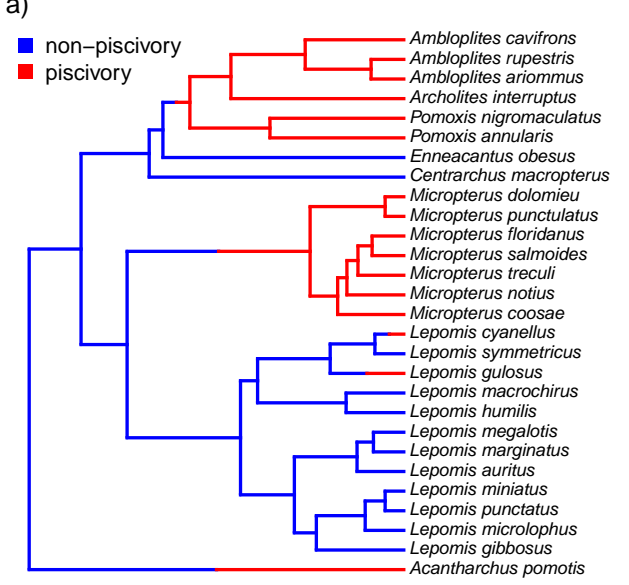

b)

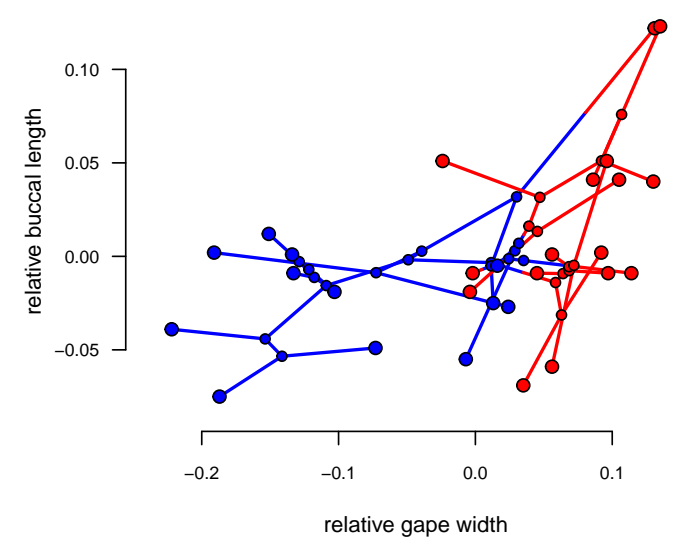

c) Fitted one-matrix model:
\[ R=\left[\begin{array}{ll}0.114 & 0.033 \\ 0.033 & 0.056\end{array}\right], \quad \log (\mathrm{L})=72.19 \]
Fitted two-matrix model:
$\quad R_{\text {non-piscivorous }}=\left[\begin{array}{ll}0.139 & -0.002 \\ -0.002 & 0.012\end{array}\right], \quad R_{\text {piscivorous }}=\left[\begin{array}{ll}0.076 & 0.080 \\ 0.080 & 0.129\end{array}\right], \quad \log (\mathrm{L})=81.24$

Figure 1. a) Phylogeny of centrarchid fishes with feeding mode (piscivory or non-piscivory) mapped onto the edges of the tree. b) Projection of the tree in (a) into a phenotypic trait space defined by different aspects of the mouth morphology in Centrarchidae. c) Fitted two-matrix evolutionary model. The evolutionary covariance between relative gape width and buccal length is higher in piscivorous compared to non-piscivorous fishes, and this model fits significantly better than a model in which the evolutionary covariance is assumed to be equal for the two regimes. Note that although this analysis is similar to the one that accompanied Revell and Collar (2009), here we've used a slightly different set of taxa and a different mapping of regimes onto the phylogeny. The phylogenetic tree is modified from Near et al. (2005).

The simpler of these two models, with only one value for the evolutionary variance-covariance matrix of the Brownian process, contains a total of five parameters to be estimated: $\sigma_{1}^{2}$ and $\sigma_{2}^{2}$ for the two traits; $\sigma_{1,2}$, the evolutionary covariance (or, in the equivalent reparameterization given by equation (3), $r$ ); and ancestral values at the root node for each trait (O'Meara et al., 2006; Hohenlohe and Arnold, 2008; Revell and Collar, 2009). By contrast, the more complex model of Figure 1c contains a total of eight estimated parameters: $\sigma_{1}^{2}, \sigma_{2}^{2}, \sigma_{1,2}$ for each of two modeled regimes (non-piscivory and piscivory), plus two ancestral states at the root.

Based on an approximately 8.1 log-likelihood unit difference between the two fitted models of our example (Figure 1), we would conclude that the two-matrix model significantly better explains the trait data than a model in which the evolutionary rates (variances) and covariances are constant across all the edges and clades of the phylogeny $(P<0.001$; Revell and Collar, 2009). We obtain a similar result if we use information theoretic model selection (such as the Akaike Information Criterion, AIC, see below; Akaike, 1974) instead of likelihood-ratio hypothesis testing. Looking specifically at the evolutionary correlation ( $r$ ), based on equation (2), above, we estimate that the correlation changes from being very slightly negative ( $r=-0.05$ ) in non-piscivorous taxa, to quite strongly positive in their piscivorous kin $(r=0.80)$. This is consistent with stronger selection for functional integration of the different elements of the feeding apparatus in piscivorous vs. non-piscivorous lineages (Collar et al., 2005; Revell and Collar, 
Table 1. Model description, model parameter estimates, $\log$-likelihood, $\log (L)$, and AIC for one homogeneous and seven heterogeneous rate or correlation multivariate Brownian evolution models fit to the data of Figure 1. $\sigma_{i, j}^{2}$ gives the instantaneous variance of the Brownian process (evolutionary rate) for the $i$ th trait and $j$ th regime. (Note that this is a different use of subscripts as compared to equation (1) in which only traits, and not regimes, were being indicated.) $r_{j}$ gives the evolutionary correlation between traits 1 and 2 for evolutionary regime $j$. In the table, regime 1 is non-piscivory and regime 2 is piscivorous feeding mode; while trait 1 is relative gape width and trait 2 is relative buccal length (Figure 1). The best-supported model using AIC as our model selection criterion (highlighted in bold font) is model 3c: different rates for trait 2, different correlations.

\begin{tabular}{lccrrrrrr} 
Model description & $\sigma_{1,1}^{2}$ & $\sigma_{1,2}^{2}$ & $\sigma_{2,1}^{2}$ & $\sigma_{2,2}^{2}$ & $\mathrm{r}_{1}$ & $\mathrm{r}_{2}$ & $\log (L)$ & $\mathrm{AIC}$ \\
\hline $\begin{array}{l}\text { common rates, } \\
\text { common correlation [1] }\end{array}$ & 0.11 & - & 0.056 & - & 0.41 & - & 72.2 & -134.4 \\
& & & & & & & & \\
\hline $\begin{array}{l}\text { different rates, } \\
\text { common correlation }\end{array}$ & 0.18 & 0.05 & 0.02 & 0.09 & 0.45 & - & 78.0 & -142.0 \\
& & & & & & & & \\
\hline $\begin{array}{l}\text { different rates (trait 1), } \\
\text { common correlation [2b] }\end{array}$ & 0.20 & 0.04 & 0.06 & - & 0.55 & - & 76.0 & -140.0 \\
\hline $\begin{array}{l}\text { different rates (trait 2), } \\
\text { common correlation [2c] }\end{array}$ & 0.11 & - & 0.02 & 0.10 & 0.33 & - & 75.3 & -138.7 \\
\hline $\begin{array}{l}\text { common rates, } \\
\text { different correlation [3] }\end{array}$ & 0.10 & - & 0.06 & - & 0.16 & 0.68 & 73.6 & -135.2 \\
\hline $\begin{array}{l}\text { different rates (trait 1), } \\
\text { different correlation [3b] }\end{array}$ & 0.17 & 0.05 & 0.06 & - & 0.36 & 0.65 & 76.5 & -139.0 \\
\hline $\begin{array}{l}\text { different rates (trait 2), } \\
\text { different correlation [3c] }\end{array}$ & $\mathbf{0 . 1 1}$ & - & $\mathbf{0 . 0 1}$ & $\mathbf{0 . 1 6}$ & $\mathbf{0 . 0 0}$ & $\mathbf{0 . 8 5}$ & $\mathbf{8 0 . 7}$ & $\mathbf{- 1 4 7 . 4}$ \\
\hline \begin{tabular}{l} 
no common structure [4] \\
\hline
\end{tabular} & 0.14 & 0.08 & 0.01 & 0.13 & -0.05 & 0.80 & 81.2 & -146.5 \\
\hline
\end{tabular}

This analysis is implemented in the phytools (Revell, 2012) software library for the R statistical computing environment (R Core Team, 2021).

\section{METHODS AND RESULTS}

\section{A hierarchical set of models}

One obvious limitation of the approach illustrated in Figure 1 is that it only considers two possible the evolutionary variance-covariance matrix among traits: one in which both the the evolutionary correlation are constant; and a second in which the two possible to identify a number of different alternative models between these two extremes.

Table 1 lists a total of eight alternative models (our original two models, from above, and six others). In square parentheses after each model, we've also provided the alphanumeric code that's been used to denominate the different models in the phytools (Revell, 2012) R software function evolvcv.lite where these models have been implemented.

The eight models of Table 1 are as follows: model (1) common rates, common correlation; model (2) different rates, common correlation; model (2b) different rates for trait 1 only, common correlation; model (2c) different rates for trait 2 only, common correlation; model (3) common rates, different correlation; 
model (3b) different rates for trait 1 only, different correlation; model (3c) different rates for trait 2 only, different correlation; finally, model (4) no common structure between the two different evolutionary variance-covariance matrices of the multivariate Brownian process.

When we analyze this complete set of models for our centrarchid dataset of Figure 1, we find that the best fitting model (that is, the model with the highest log-likelihood) is the no common structure model in which the Brownian evolutionary variance-covariance matrix is free to differ in all possible ways depending on the mapped regime. It is, in fact, a logical necessity that model 4 has a log-likelihood that's greater than or equal to the next best model. This is because model 4, our no common structure model, has all of our other seven models as special cases. On the other hand, the best supported model (that is, the model that's best-justified by our data taking into account model complexity; Burnham and Anderson, 2002) is model 3c (different rates in trait 2, relative buccal length, different correlations; Table 1 ), indicated with bold font in the table.

Note that some other software, such as the mvMORPH R package of Clavel et al. (2015), also fits alternative models for multivariate Brownian evolution - such as a model in which the rate of evolution for different traits or for different regimes are constrained to be equal, or a model in which the evolutionary correlation between traits, $r$, is constrained to be 0 .

\section{An empirical example: South American rock- and non rock-dwelling lizards}

In addition to the centrarchid data, above, we also applied the method to a morphological dataset of South American iguanian lizards (members of the lizard family Tropiduridae sensu lato; Toyama, 2017). For this example, we mapped habitat use of rock-dwelling vs. non rock-dwelling (Revell et al., 2007; Goodman et al., 2008) on a phylogeny of 76 lizard species. Our phylogeny was obtained from Pyron et al. (2013), but pruned to contain only the taxa of the present study, and rescaled to have a total length of 1.0. (We rescaled the tree to unit length merely so that our parameter wouldn't need to be represented using scientific notation. Relative model fits should be completely unaffected by this rescaling.)

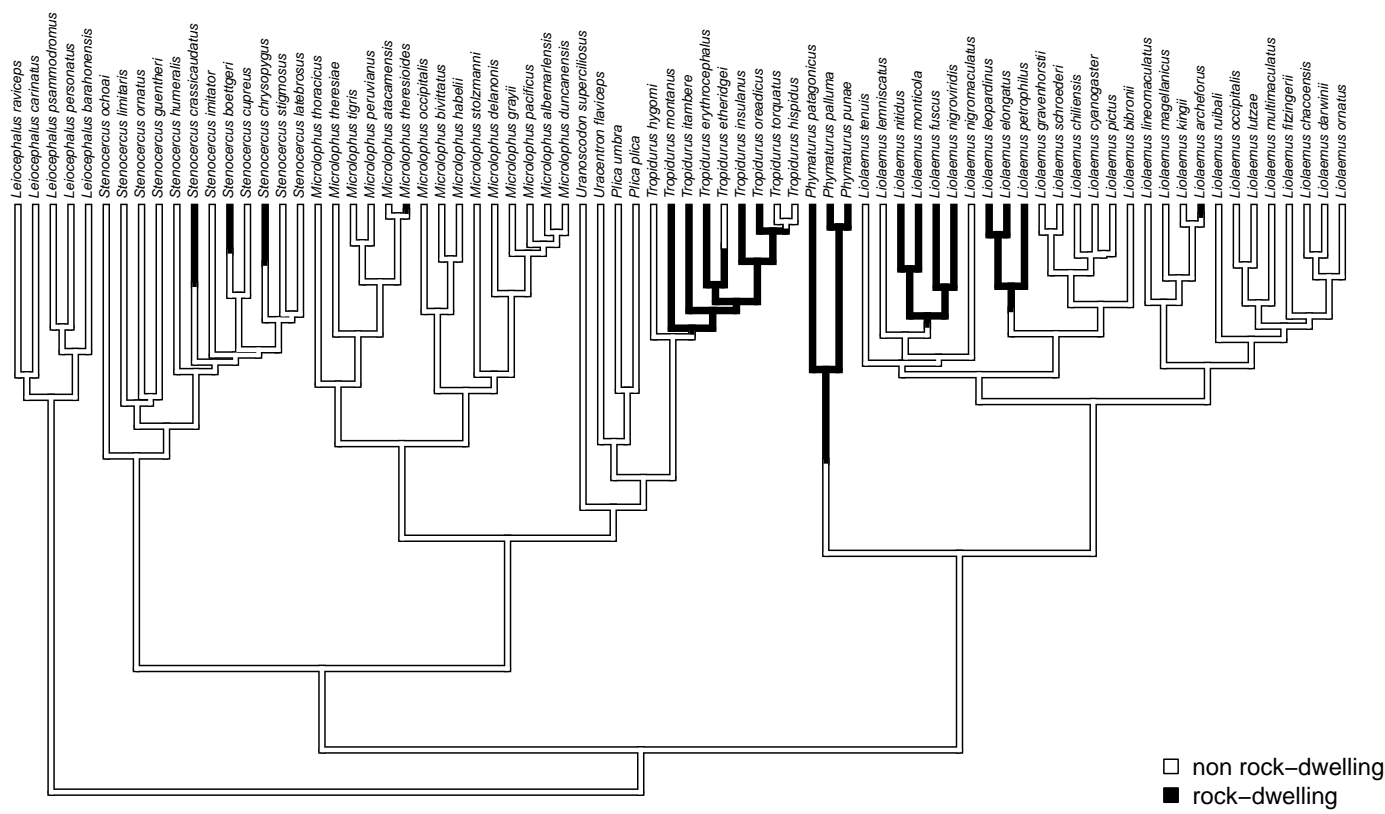

Figure 2. Phylogenetic tree of 76 South American iguanian lizards species based on Pyron et al. (2013). Colors indicate two different mapped ecological regimes: rock-dwelling (in black) and non rock-dwelling (white). The tree has been rescaled to have a total depth of 1.0.

To set our regimes, we used a single Maximum Parsimony reconstruction of the discrete trait (rockvs. non rock-dwelling) on our phylogeny, in which we fixed all transitions between regimes to be located at the precise midpoint of each edge containing a state change in our reconstruction. In an empirical study, we would probably recommend using multiple reconstructions from a statistical method such as 
stochastic character mapping (Huelsenbeck et al., 2003), and then averaging the results across stochastic maps (e.g., O'Meara et al., 2006; but see Revell, 2013). Our lizard phylogeny with mapped regimes is shown in Figure 2.

We next fit all eight of the models listed in Table 1 to a dataset consisting of body size and relative body dorsoventral depth from Toyama (2017), both calculated using geometrical definitions for size and shape (Mosimann, 1970; Klingenberg, 2016). Since rock-dwelling has previously been suggested to favor the evolution of dorsoventral flattening (e.g., Revell et al., 2007; Goodman et al., 2008), we hypothesized that the evolutionary correlation between body size and depth, while generally positive across this group, could decrease or become negative in rock-dwelling forms due to ecological selection to decouple body depth from size. In Table 2, we show the parameter estimates, model fits, and Akaike weights (see section below) of the top-four best-supported models from this analysis.

Table 2. Model rank, model name, model parameter estimates, $\log$-likelihood, $\log (L)$, AIC, and Akaike weights for the top four heterogeneous rate or correlation multivariate Brownian evolution models fit to overall size and relative body depth in South American iguanian lizards (Figure 2). Column headers are as in Table 1, except for $w$, which indicates Akaike weight as calculated using equation (4).

\begin{tabular}{clrrrrrrrrr}
\hline Rank & Model & $\sigma_{1,1}^{2}$ & $\sigma_{1,2}^{2}$ & $\sigma_{2,1}^{2}$ & $\sigma_{2,2}^{2}$ & $\mathrm{r}_{1}$ & $\mathrm{r}_{2}$ & $\log (L)$ & AIC & $w$ \\
\hline 1 & model 3 & 0.23 & - & 0.06 & - & 0.34 & -0.31 & 55.11 & -98.22 & 0.28 \\
2 & model 3c & 0.23 & - & 0.05 & 0.10 & 0.33 & -0.31 & 56.04 & -98.08 & 0.26 \\
3 & model 3b & 0.21 & 0.27 & 0.06 & - & 0.33 & -0.32 & 55.32 & -96.63 & 0.13 \\
4 & model 4 & 0.21 & 0.28 & 0.05 & 0.10 & 0.32 & -0.34 & 56.29 & -96.58 & 0.12 \\
\hline
\end{tabular}

Although the weight of evidence is distributed among our top four models in the table, the most notable aspect of all of the best-supported models for these data is that they each allow the evolutionary correlation $(r)$ to differ between the two different mapped regimes on the tree. Models that don't allow the evolutionary correlation to different by regime (models $1,2,2 \mathrm{~b}$, and $2 \mathrm{c}$ from Table 1 ) each received less than $10 \%$ support.

We also found that the evolutionary correlation between body size and size-adjusted body depth was positive in non rock-dwelling lizards, indicating that larger lizards tended to evolve proportionally greater body depth (Table 2). By contrast, rock-dwelling forms actually exhibited a negative evolutionary correlation between body size and size-adjusted body depth. This is because larger rock-dwelling animals do not tend to evolve proportionally greater body depths. To the contrary, their size-adjusted body depth actually decreases. This is largely consistent with what's expected given behavioral and biomechanical considerations (Revell et al., 2007; Goodman et al., 2008).

\section{A small simulation test of the method}

In addition to the empirical applications given above, we tested the method using a small simulation experiment as follows. We first generated twenty 100-taxon pure-birth random phylogenetic trees. On each of these trees, we simulated the history of a three-state discrete character. We rejected and repeated any simulation in which any of the three states of the trait was not observed in at least twenty tips. An example simulated tree with evolutionary regimes is given in Figure 3a.

For all of the twenty random trees, we simulated data under each of the eight models of Table 1 . To begin each simulation, we first drew values for $\log \left(\sigma_{1}^{2}\right)$ and $\log \left(\sigma_{2}^{2}\right)$ for the two traits from a standard normal distribution (that is to say, $\sigma_{1}^{2}$ and $\sigma_{2}^{2}$ were randomly sampled from a log-normal distribution); and we drew a random value or values of the correlation coefficient $(r)$ from a uniform distribution on the interval -1 to 1 . Naturally, we sampled different numbers of values for $\log \left(\sigma_{1}^{2}\right), \log \left(\sigma_{2}^{2}\right)$, and $r$ depending on the model that was being used for simulation. For instance, a model with three mapped regimes (e.g., Figure 3a) and different rates for trait 1, equal rates for trait 2 , and different correlations between traits 1 and 2, would involve randomly sampling three values for $\log \left(\sigma_{1}^{2}\right)$, one value for $\log \left(\sigma_{2}^{2}\right)$, and three values for $r$ from their respective distributions. Our simulation procedure doesn't fix any specific difference in the rates or evolutionary correlations between regimes. Nonetheless, it will on average result in a geometric mean ratio of the highest evolutionary rate over the lowest (for any variable $\sigma^{2}$ simulation) of around 5.4; and a mean difference between the highest evolutionary correlation and the lowest (for any variable $r$ simulation) of about 1.0. 
a)

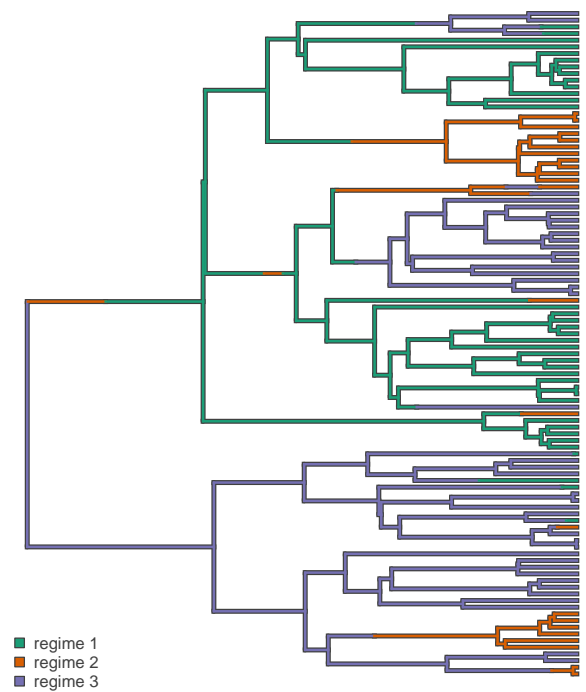
$3 b)$.

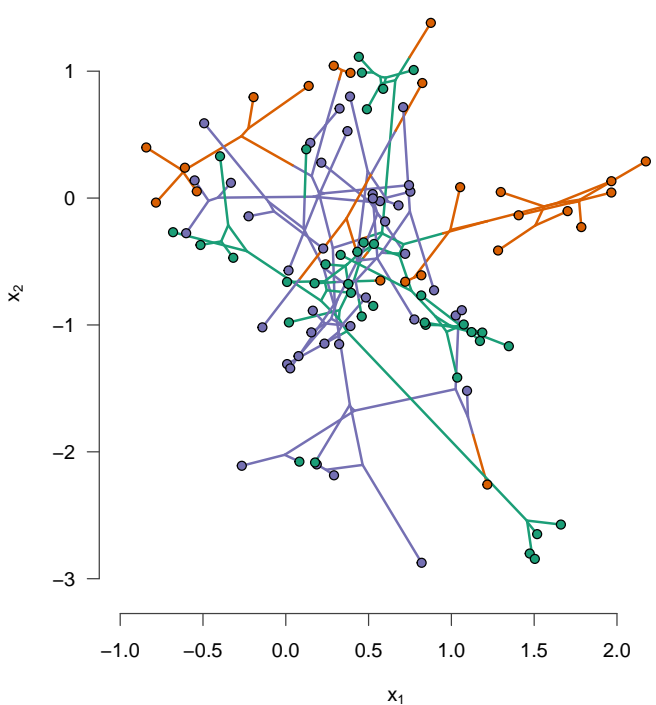

Figure 3. a) Example simulated phylogenetic tree with three mapped evolutionary regimes. b) The phylogeny of panel (a) projected into a two dimensional phenotypic trait space. The trait data in panel (b) were simulated under model $3 \mathrm{~b}$ from Table 1 (different rates in trait 1 , different correlations), in which the simulated evolutionary correlation between traits $x_{1}$ and $x_{2}$ was negative in regimes 1 and 3 , but positive in regime 2.

An example simulated dataset generated using our procedure for different rates (trait 1), and different correlations (model 3b) is shown in Figure 3b. In this example, we simulated the data using an evolutionary correlation between traits $x_{1}$ and $x_{2}$ that was negative for regimes 1 and 3, but positive for regime 2 (Figure

After completing the numerical simulations, we then proceeded to fit each of the same eight models to each simulated dataset. For each fitted model, we computed AIC and Akaike weights as follows (Akaike, 1974; Burnham and Anderson, 2002).

$$
A I C_{i}=2 k-2 \ln \left(l_{i}\right) \quad w_{i}=\frac{e^{-\Delta A I C_{i} / 2}}{\Sigma e^{-\Delta A I C_{j} / 2}}
$$

Here, $A I C_{i}$ is the value of AIC for the $i$ th model; $k$ is the number of parameters in the model; $\ln \left(l_{i}\right)$ is the log-likelihood of the $i$ th model; and $\triangle A I C_{i}$ is the difference in AIC between the $i$ th model and the model with the minimum AIC score in the set. In general, we should prefer the model with the lowest overall value for AIC, and can interpret the Akaike model weights ( $w$ ), from equation (4), as a measure of the strength of evidence supporting each of the models in our set (Akaike, 1974; Wagenmakers and Farrell, 2004).

After fitting all eight models to each of our $20 \times 8=160$ simulated datasets, we next simply calculated the fraction of times in which the generating model was selected as the 'best' model (as well as second best, third best, and so on). These results are summarized in Table 3. In general, we found that the generating model tended to be selected as the best or second best model over $86 \%$ of the time in simulation, under the simulation conditions described above (Table 3).

In addition, we also calculated the average weight $(\bar{w})$ of each of the twenty datasets for each model. These results are summarized in Figure 4. This analysis shows that the generating model (in rows) also tended to have the highest average Akaike model weight (in columns; Figure 4). 
Table 3. Model name and the fraction of times from twenty simulations in which the generating model was identified as the best, 2nd best, 3rd best, or worse than third best model using AIC model selection.

\begin{tabular}{lrrrr} 
Model name & Best & 2nd best & 3rd best & $\geq$ 4th \\
\hline model 1 & 0.65 & 0.15 & 0.05 & 0.15 \\
model 2 & 0.70 & 0.20 & 0.05 & 0.05 \\
model 2b & 0.65 & 0.10 & 0.15 & 0.10 \\
model 2c & 0.80 & 0.10 & 0.10 & 0.00 \\
model 3 & 0.75 & 0.15 & 0.05 & 0.05 \\
model 3b & 0.75 & 0.15 & 0.05 & 0.05 \\
model 3c & 0.40 & 0.35 & 0.20 & 0.05 \\
model 4 & 0.65 & 0.35 & 0.00 & 0.00 \\
\hline
\end{tabular}

For each generating model, the next highest average Akaike model weights tended to be observed in models of similar complexity. For instance, when the generating model was model 4 (no common structure), we found the highest average model weight for model 4 (0.65); and then the next highest average model weights for models $3 b$ (different rates for trait 1 , different correlations; 0.14 ) and $3 c$ (different rates for trait 2, different correlations; 0.13). Conversely, when the generating model was model 1 (common rates, common correlation), we found the highest average model weight for model $1(0.37)$, and the next highest average model weight for model 3 (common rates, different correlation; 0.14).

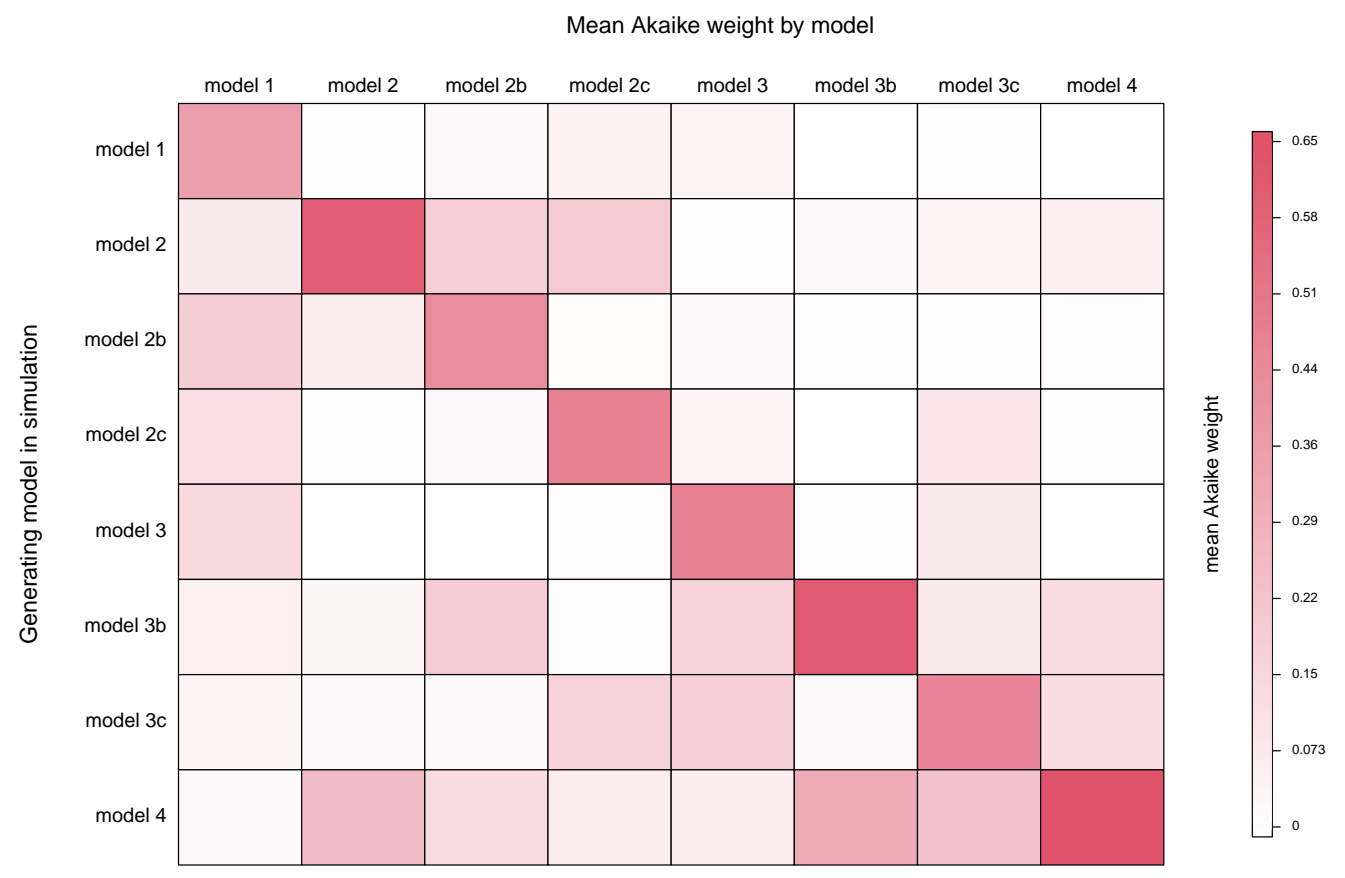

Figure 4. Mean Akaike weight for all eight models (in columns) for each of the eight generating models (in rows). Simulation conditions were as described in the text.

\section{Notes on implementation}

The model and methods of this study have been implemented for the R statistical computing environment (R Core Team, 2021), and all simulations and analyses for this study were done using R.

The method that we describe in the article is implemented as the function evolvcv.lite of the phytools $\mathrm{R}$ package (Revell, 2012). phytools in turn depends on the important $\mathrm{R}$ phylogenetics packages ape (Paradis and Schliep, 2019) and phangorn (Schliep, 2011), as well as on a number of other R libraries 
(Venables and Ripley, 2002; Ligges and Mächler, 2003; Lemon, 2006; Plummer et al., 2006; Chasalow, 2012; Becker et al., 2018; Gilbert and Varadhan, 2019; Azzalini and Genz, 2020; Qiu and Joe, 2020; Warnes et al., 2020; Goulet et al., 2021; Pinheiro et al., 2021).

\section{DISCUSSION}

The evolutionary correlation is defined as the tendency of two different phenotypic traits to co-evolve (Harmon, 2019). Traits are said to have a positive evolutionary correlation if a large increase in the value of one trait tends to be a accompanied by a similarly large increase in the second, and vice versa. Traits can be evolutionarily correlated for a wide variety of reasons. For instance, a genetic correlation between traits, if persistent over macroevolutionary time periods, will tend to cause two phenotypic characteristics to evolve in a correlated fashion, even under genetic drift (Schluter, 1996; Blows and Hoffmann, 2005; Hohenlohe and Arnold, 2008; Revell and Harmon, 2008). Genetic correlations between traits in turn tend to be causes by pleiotropy, such as when one quantitative trait locus affects the expressed value of two different phenotypic attributes (e.g., Gardner and Latta, 2007).

More often, however, when an evolutionary correlation between traits is observed, natural selection tends to be purported. For instance, the evolutionary correlation between water-related plant traits observed by Sun et al. (2020) was interpreted by the authors as evidence for natural selection acting to favor certain combinations of trait values over others. Likewise, when Goodwillie et al. (2009) found an evolutionary correlation between reproductive outcrossing rate and the product of flower number and size in plants, they hypothesized that this was due to selection favoring increased investment in structures to attract pollinators in outcrossing compared to selfing taxa. Numerous questions in evolutionary research involve measuring the evolutionary correlations between traits (Felsenstein, 1985; Harmon, 2019), and in many cases it may be sufficient to fit a single value of the evolutionary correlation between characters for all the branches and nodes of the phylogeny. Under other circumstances, however, it's useful or necessary to permit the evolutionary correlation to assume different values in different parts of the tree.

For example, in the present study we used data for centrarchid fishes to test whether feeding mode influences the evolutionary tendency of two different aspects of the buccal morphology to co-evolve (Revell and Collar, 2009). We hypothesized that natural selection for functional integration of the feeding apparatus constrains different buccal traits to evolve in a coordinated fashion in piscivorous lineages, but not in their non-piscivorous kin (Collar et al., 2005). Indeed, our analysis reiterates the finding of Revell and Collar (2009) in showing that a model with different evolutionary correlations between traits depending on feeding mode significantly better explains our morphological trait data, compared to a model in which the evolutionary correlation is forced to have a constant value across all the branches of the phylogeny. Like Revell and Collar (2009), we also found that the evolutionary correlation between buccal traits is high and positive in piscivorous but not non-piscivorous lineages (Table 1). Unlike Revell and Collar (2009), however, we found that the best-supported model was one in which the evolutionary rate $\left(\sigma^{2}\right)$ for buccal length, but not gape width, was also free to differ in different parts of the phylogeny.

Likewise, we present data for the evolution of overall body size and size-adjusted dorsoventral body depth in South American iguanian rock-dwelling and non rock-dwelling lizards, a group rich in habitat transitions (Figure 2; Toyama, 2017). Based on prior research (Revell et al., 2007; Goodman et al., 2008), we hypothesized that selection might favor the decoupling of a normally positive evolutionary correlation between the two traits to permit the evolution of greater dorsoventral compression in rock-dwelling species. Indeed, all four of the best-fitting models in our analysis were ones in which the evolutionary correlation was permitted to differ by habitat use: rock or non-rock (Table 2). Models where the evolutionary rates $\left(\sigma^{2}\right)$, but not the evolutionary correlation $(r)$, differed across the tree received very little support.

Finally, we undertook a small simulation study of our method. We found that the generating model in simulation also tended to be the model that was most often chosen via our model selection procedure (Table 3; Figure 4). When the generating model was not best-supported, a model of similar parameterization tended to be selected instead (Figure 4).

\section{Relationship to other methods}

Readers of this article who are familiar with phylogenetic comparative methods might observe that it's also possible to model multivariate trait evolution in which the relationship between traits changes as a function of a discrete factor using a phylogenetic generalized analysis of covariance (Grafen, 1989; Rohlf, 2001; Revell, 2010; Mundry, 2014; Fuentes-G. et al., 2016). 
In this case, we'd simply fit a linear model in which a single dependent variable $(y)$ varied as a function of a discrete factor (the tip regime), a continuous variable $(x)$, and their interaction (to permit differences in slope between regimes), while assuming that the residual error in $y$ has a multivariate normal distribution given by the structure of the tree (Rohlf, 2001; Revell, 2010; Fuentes-G. et al., 2016). Indeed, this is a valid approach for asking how the relationship between traits changes among lineages of a reconstructed phylogeny. We nonetheless feel that our method adds value for many investigators because it permits an arbitrary (not just tip) mapping of discrete regimes, because it doesn't require the user to specify dependent and independent variables in the model, because it easily allows us to take into account sampling error in the estimation of species' means (following Ives et al., 2007), because it's readily extensible to more than two traits whose correlations might also be expected to change as a function of the mapped regimes, and, finally, because it's more directly connected to a hypothesized evolutionary process for the traits on our phylogeny (Hohenlohe and Arnold, 2008; Revell and Harmon, 2008).

\section{Conclusions}

The evolutionary correlation is defined as the tendency for changes in one phenotypic attribute to be associated (positively or negatively) with changes in a second trait through evolutionary time or on a phylogenetic tree (Harmon, 2019). Many questions in phylogenetic comparative biology involve measuring the evolutionary correlations between characters using phylogenies. Often, it's sufficient to assume a constant value of this evolutionary correlation through time or among clades. Here, however, we present a hierarchical series of models in which we permit the rate of evolution for traits, and their evolutionary correlation, to differ in different parts of the phylogeny that have been specified a priori by the investigator.

\section{ACKNOWLEDGMENTS}

This research was funded by grants from the National Science Foundation (DBI-1759940) and FONDECYT, Chile (1201869), to LJR; and a Natural Sciences and Engineering Research Council of Canada Discovery Grant (RGPIN-2015-04334), to DLM.

\section{REFERENCES}

Adams, D. C. (2013). Comparing evolutionary rates for different phenotypic traits on a phylogeny using likelihood. Systematic Biology, 62(2):181-192.

Akaike, H. (1974). A new look at the statistical model identification. IEEE Transactions on Automatic Control, 19(6):716-723.

Azzalini, A. and Genz, A. (2020). The R package mnormt: The multivariate normal and $t$ distributions. $\mathrm{R}$ package verison 2.0.2.

Becker, R. A., Wilks, A. R., Brownrigg, R., Minka, T. P., and Deckmyn, A. (2018). maps: Draw Geographical Maps. R package version 3.3.0.

Blows, M. W. and Hoffmann, A. A. (2005). A reassessment of genetic limits to evolutionary change. Ecology, 86(6):1371-1384.

Burnham, K. P. and Anderson, D. R. (2002). Model selection and multimodel inference : a practical information-theoretic approach. Springer, New York.

Caetano, D. S. and Harmon, L. J. (2017). ratematrix: An R package for studying evolutionary integration among several traits on phylogenetic trees. Methods in Ecology and Evolution, 8(12):1920-1927.

Chasalow, S. (2012). combinat: combinatorics utilities. R package version 0.0-8.

Clavel, J., Escarguel, G., and Merceron, G. (2015). mvMORPH: an R package for fitting multivariate evolutionary models to morphometric data. Methods in Ecology and Evolution, 6:1311-1319.

Collar, D. C., Near, T. J., and Wainwright, P. C. (2005). Comparative analysis of morphological diversity: Does disparity accumulate at the same rate in two lineargs of centrarchid fishes? Evolution, 59(8):17831794.

Damian-Serrano, A., Haddock, S. H. D., and Dunn, C. W. (2021). The evolution of siphonophore tentilla for specialized prey capture in the open ocean. Proceedings of the National Academy of Sciences, 118(8):e2005063118.

Felsenstein, J. (1985). Phylogenies and the comparative method. The American Naturalist, 125(1):1-15. 
Fuentes-G., J. A., Housworth, E. A., Weber, A., and Martins, E. P. (2016). Phylogenetic ANCOVA: Estimating changes in evolutionary rates as well as relationships between traits. The American Naturalist, 188(6):615-627.

Gardner, K. M. and Latta, R. G. (2007). Shared quantitative trait loci underlying the genetic correlation between continuous traits. Molecular Ecology, 16(20):4195-4209.

Garland, T., Harvey, P. H., and Ives, A. R. (1992). Procedures for the analysis of comparative data using phylogenetically independent contrasts. Systematic Biology, 41(1):18-32.

Gilbert, P. and Varadhan, R. (2019). numDeriv: Accurate Numerical Derivatives. R package version 2016.8-1.1.

Goodman, B. A., Miles, D. B., and Schwarzkopf, L. (2008). Life on the rocks: habitat use drives morphological and performance evolution in lizards. Ecology, 89(12):3462-3471.

Goodwillie, C., Sargent, R. D., Eckert, C. G., Elle, E., Geber, M. A., Johnston, M. O., Kalisz, S., Moeller, D. A., Ree, R. H., Vallejo-Marin, M., and Winn, A. A. (2009). Correlated evolution of mating system and floral display traits in flowering plants and its implications for the distribution of mating system variation. New Phytologist, 185(1):311-321.

Goulet, V., Dutang, C., Maechler, M., Firth, D., Shapira, M., and Stadelmann, M. (2021). expm: Matrix Exponential, Log, 'etc'. R package version 0.999-6.

Grafen, A. (1989). The phylogenetic regression. Philosophical Transactions of the Royal Society of London. B, Biological Sciences, 326(1233):119-157.

Harmon, L. (2019). Phylogenetic Comparative Methods. Independent, Open Textbook Library, Minneapolis.

Hohenlohe, P. A. and Arnold, S. J. (2008). MIPoD: A hypothesis-testing framework for microevolutionary inference from patterns of divergence. The American Naturalist, 171(3):366-385.

Huelsenbeck, J. P., Nielsen, R., and Bollback, J. P. (2003). Stochastic mapping of morphological characters. Systematic Biology, 52(2):131-158.

Ives, A. R., Midford, P. E., and Garland, T. (2007). Within-species variation and measurement error in phylogenetic comparative methods. Systematic Biology, 56(2):252-270.

Klingenberg, C. P. (2016). Size, shape, and form: concepts of allometry in geometric morphometrics. Development Genes and Evolution, 226(3):113-137.

Lemon, J. (2006). Plotrix: a package in the red light district of r. R-News, 6(4):8-12.

Ligges, U. and Mächler, M. (2003). Scatterplot3d - an r package for visualizing multivariate data. Journal of Statistical Software, 8(11):1-20.

Mosimann, J. E. (1970). Size allometry: Size and shape variables with characterizations of the lognormal and generalized gamma distributions. Journal of the American Statistical Association, 65(330):930945.

Mundry, R. (2014). Statistical issues and assumptions of phylogenetic generalized least squares. In Modern Phylogenetic Comparative Methods and Their Application in Evolutionary Biology, pages 131-153. Springer Berlin Heidelberg.

Near, T. J., Bolnick, D. I., and Wainwright, P. C. (2005). Fossil calibrations and molecular divergence time estimates in centrarchid fishes (Teleostei: Centrarchidae). Evolution, 59(8):1768-1782.

O'Meara, B. C. (2012). Evolutionary inferences from phylogenies: A review of methods. Annual Review of Ecology, Evolution, and Systematics, 43(1):267-285.

O'Meara, B. C., Ané, C., Sanderson, M. J., and Wainwright, P. C. (2006). Testing for different rates of continuous trait evolution using likelihood. Evolution, 60(5):922-933.

Paradis, E. and Schliep, K. (2019). ape 5.0: an environment for modern phylogenetics and evolutionary analyses in R. Bioinformatics, 35:526-528.

Pinheiro, J., Bates, D., DebRoy, S., Sarkar, D., and R Core Team (2021). nlme: Linear and Nonlinear Mixed Effects Models. R package version 3.1-152.

Plummer, M., Best, N., Cowles, K., and Vines, K. (2006). CODA: Convergence diagnosis and output analysis for memc. $R$ News, 6(1):7-11.

Pyron, R., Burbrink, F. T., and Wiens, J. J. (2013). A phylogeny and revised classification of squamata, including 4161 species of lizards and snakes. BMC Evolutionary Biology, 13(1):93.

Qiu, W. and Joe, H. (2020). clusterGeneration: Random Cluster Generation (with Specified Degree of Separation). R package version 1.3.7.

$\mathrm{R}$ Core Team (2021). R: A Language and Environment for Statistical Computing. R Foundation for 
Statistical Computing, Vienna, Austria.

Revell, L. J. (2010). Phylogenetic signal and linear regression on species data. Methods in Ecology and Evolution, 1(4):319-329.

Revell, L. J. (2012). phytools: an r package for phylogenetic comparative biology (and other things). Methods in Ecology and Evolution, 3(2):217-223.

Revell, L. J. (2013). A comment on the use of stochastic character maps to estimate evolutionary rate variation in a continuously valued trait. Systematic Biology, 62(2):339-345.

Revell, L. J. (2021). A variable-rate quantitative trait evolution model using penalized-likelihood.

Revell, L. J. and Collar, D. C. (2009). Phylogenetic analysis of the evolutionary correlation using likelihood. Evolution, 63(4):1090-1100.

Revell, L. J. and Harmon, L. J. (2008). Testing quantitative genetic hypotheses about the evolutionary rate matrix for continuous characters. Evolutionary Ecology Research, 10:311-331.

Revell, L. J., Harmon, L. J., and Collar, D. C. (2008). Phylogenetic signal, evolutionary process, and rate. Systematic Biology, 57(4):591-601.

Revell, L. J., Johnson, M. A., Schulte, J. A., Kolbe, J. J., and Losos, J. B. (2007). A phylogenetic test for adaptive convergence in rock-dwelling lizards. Evolution, 61(12):2898-2912.

Rohlf, F. J. (2001). Comparative methods for the analysis of continuous variables: Geometric interpretations. Evolution, 55(11):2143-2160.

Ruiz-Robleto, J. and Villar, R. (2005). Relative growth rate and biomass allocation in ten woody species with different leaf longevity using phylogenetic independent contrasts (PICs). Plant Biology, 7(5):484-494.

Schliep, K. P. (2011). phangorn: phylogenetic analysis in r. Bioinformatics, 27(4):592-593.

Schluter, D. (1996). Adaptive radiation along genetic lines of least resistance. Evolution, 50(5):1766.

Sun, M., Feng, C.-H., Liu, Z.-Y., and Tian, K. (2020). Evolutionary correlation of water-related traits between different structures of dendrobium plants. Botanical Studies, 61(1).

Thomas, G. H., Freckleton, R. P., and Székely, T. (2006). Comparative analyses of the influence of developmental mode on phenotypic diversification rates in shorebirds. Proceedings of the Royal Society B: Biological Sciences, 273(1594):1619-1624.

Toyama, K. S. (2017). Interaction between morphology and habitat use: A large-scale approach in tropidurinae lizards. Breviora, 554(1):1.

Venables, W. N. and Ripley, B. D. (2002). Modern Applied Statistics with S. Springer, New York, fourth edition. ISBN 0-387-95457-0.

Wagenmakers, E.-J. and Farrell, S. (2004). AIC model selection using akaike weights. Psychonomic Bulletin \& Review, 11(1):192-196.

Warnes, G. R., Bolker, B., and Lumley, T. (2020). gtools: Various R Programming Tools. R package version 3.8.2. 